\title{
Herança de caracteres associados à eficiência de utilização do fósforo em milho
}

\author{
Roberto Fritsche-Neto(1), Glauco Vieira Miranda(1), Rodrigo Oliveira DeLima(1), \\ Leandro Vagno de Souza(2) e Jaeveson da Silva ${ }^{(3)}$
}

\begin{abstract}
(1)Universidade Federal de Viçosa, Departamento de Fitotecnia, CEP 36570-000 Viçosa, MG. E-mail: rfritscheneto@gmail.com, glaucovmiranda@ufv.br, rodrigoodelima@gmail.com ${ }^{(2)}$ Dow Agrosciences Industria Ltda., Rodovia Anhanguera, s/no, Km 344, Zona Rural, CEP14680-000 Ribeirão Preto, SP. E-mail: Ivsouza2@dow.com ${ }^{(3)}$ Embrapa Mandioca e Fruticultura, Caixa Postal 007, Rua Embrapa, s/no, Centro, CEP 44380-000 Cruz das Almas, BA. E-mail: jaeveson@enpmf.embrapa.br
\end{abstract}

Resumo - O objetivo deste trabalho foi identificar os efeitos genéticos que controlam a herança de caracteres associados à eficiência de utilização do fósforo (EUP) em milho e determinar as relações entre esses caracteres. Foi realizado um dialelo entre seis cultivares de milho do qual foram obtidas 15 combinações híbridas. Estas cultivares foram avaliadas em alta e baixa disponibilidade de fósforo, em casa de vegetação e campo. Foram medidas a resposta das plantas à disponibilidade de fósforo (RU) e as características associadas às eficiências de utilização e translocação do nutriente. As combinações híbridas apresentaram interação significativa com a disponibilidade de P, para os caracteres massa de matéria seca da planta (MSP), teor de P na planta (PAP) e razão entre as massas de matéria seca da parte aérea e da raiz (RMS). A interação entre capacidade geral de combinação e RU foi significativa para PAP e RMS e, entre capacidade específica de combinação e RU, para MSP e PAP. As EUP em baixa e alta disponibilidade do nutriente estiveram correlacionadas entre si e com RU. A EUP em baixa disponibilidade de P não se correlacionou com a produtividade de grãos. Contudo, sob alta disponibilidade do nutriente, esses parâmetros se correlacionaram. Os efeitos não aditivos têm maior importância para caracteres relacionados à EUP, de modo que a seleção deve ser realizada nas combinações híbridas.

Termos para indexação: Zea mays, capacidade de combinação, estresse abiótico, melhoramento vegetal, nutrição mineral.

\section{Inheritance of traits associated with phosphorus utilization efficiency in maize}

\begin{abstract}
The objective of this work was to determine the genetic effects that control the inheritance of characters associated with the phosphorus utilization efficiency (PUE) in maize, and to determine the relationship among them. A diallel between six maize cultivars was performed and 15 hybrid combinations were obtained from it. These cultivars were evaluated in high and low phosphorus availability in the greenhouse and field. Plant response to nutrient availability (RU) and traits associated to utilization and translocation efficiency were measured. There were significant interactions between hybrid combinations and phosphorus availability for plant dry matter mass (MSP), P concentration in plants (PAP), and the ratio between shoot and root dry matter masses (RMS). The interaction between general combining ability and P availability was significant for RMS and PAP, and the one between specific combining ability and P availability, for MSP and PAP. The PUE in low and high P were correlated with each other and with RU. The PUE at low P availability was not correlated to grain yield. However, in high soil $P$ availability these parameters were correlated. Non additive effects are more important for traits related to PUE, so that selection should be carried out on hybrid combinations.
\end{abstract}

Index Terms: Zea mays, combining ability, abiotic stress, plant breeding, mineral nutrition.

\section{Introdução}

Maior eficiência de utilização do P (EUP) pode ser obtida pelo aumento na aquisição e na utilização interna do nutriente pelas plantas (Hammond et al., 2004; Parentoni \& Souza Junior, 2008). A melhoria de EUP pelo aumento na aquisição de $\mathrm{P}$ é resultante da morfologia e arquitetura da raiz e está relacionada ao aumento do volume e área radicular. Maior EUP proporciona aumento da produtividade de grãos por unidade de P translocada (Baligar \& Fageria, 1999).

O conhecimento dos caracteres e dos mecanismos envolvidos na eficiência nutricional (aquisição, utilização e translocação de nutrientes para os grãos), assim como de sua herança genética em solos com baixos teores de nutrientes, é fundamental para orientar

Pesq. agropec. bras., Brasília, v.45, n.5, p.465-471, maio 2010 
o melhorista quanto ao método mais adequado de melhoramento a ser empregado (Gaume et al., 2001; Yun \& Kaeppler, 2001; Hirel et al., 2007). Assim, parâmetros genéticos como capacidade geral (CGC) e específica (CEC) de combinação e herdabilidade são muito importantes para se realizar inferências acerca do controle genético do caráter de interesse e sobre as possibilidades de melhoramento, bem como para indicação de novas cultivares (Oliveira Junior et al., 1999; Betrán et al., 2003; Miranda et al., 2008; Souza et al., 2008).

Em plantas, os métodos de avaliação precoce ou de seleção indireta também são de interesse no melhoramento sob condições de estresse abiótico, pois aceleram o processo de seleção e rapidamente descartam genótipos mais suscetíveis, o que resulta na concentração de recursos potencialmente superiores (Machado et al., 2004). O trabalho de Miranda et al. (2005), em que foram descartadas linhagens de milho com menor eficiência no uso de nitrogênio $(\mathrm{N})$ com base na leitura do clorofilômetro por ocasião do florescimento das plantas é um exemplo do uso de seleção indireta para melhoramento.

Os objetivos deste trabalho foram identificar os efeitos genéticos que controlam a herança de caracteres associados à eficiência de utilização do fósforo em milho, bem como determinar as relações entre esses caracteres.

\section{Material e Métodos}

Foram avaliadas seis cultivares comerciais de milho, AG 9010, BR 201, DKB 747, Sol da manhã, BR 106 e Caiano, e 15 combinações híbridas $(\mathrm{CH})$ obtidas por meio do cruzamento dialélico entre essas cultivares. As cultivares foram selecionadas considerando sua adaptação e potencial produtivo. Foram realizados dois experimentos, um em casa de vegetação e outro em condições de campo.

No experimento em casa de vegetação, sementes das $15 \mathrm{CH}$ foram plantadas em vasos opacos de polietileno contendo $3,0 \mathrm{dm}^{3}$ de solo (Argissolo Vermelho-Amarelo). De acordo com a análise química, o solo apresentou: $\mathrm{pH}, 5,92 ; \mathrm{P}, 8,4 \mathrm{mg} \mathrm{dm}^{-3}$, e K, $56 \mathrm{mg} \mathrm{dm}^{-3}$. Não foi necessária a correção da acidez, tendo-se realizado apenas a adubação com $50 \mathrm{mg} \mathrm{dm}^{-3}$ de $\mathrm{N}$, na forma de ureia, e de $40 \mathrm{mg} \mathrm{dm}^{-3} \mathrm{de}_{2} \mathrm{O}$, como cloreto de potássio. A adubação fosfatada foi localizada a 1/3 da profundidade do vaso. A umidade do solo foi mantida em torno de $70 \%$ da capacidade de campo com irrigações diárias, com base nas pesagens dos vasos.

As $15 \mathrm{CH}$ foram avaliadas em dois níveis de adubação fosfatada. No primeiro nível, não houve acréscimo de P. No segundo, acrescentou-se, na forma de superfosfato simples, $240 \mathrm{mg} \mathrm{kg}^{-1}$ de $\mathrm{P}_{2} \mathrm{O}_{5}$, dose quatro vezes maior que a recomendada pela análise do solo. O experimento foi conduzido em delineamento experimental inteiramente casualizado, em esquema fatorial 15x2 - 15 genótipos e dois níveis de $\mathrm{P}$-, com duas repetições, no Campo Experimental Prof. Diogo Alves de Mello (2046'24"S; 4252'13"W), da Universidade Federal de Viçosa, Viçosa, MG, no primeiro semestre de 2002.

No plantio, foram utilizadas dez sementes por vaso, tendo-se deixado, após o desbaste, cinco plantas. Cada vaso de polietileno foi considerado uma unidade experimental. Aos 30 dias após o plantio, as plantas foram colhidas, lavadas em água destilada, separadas em parte aérea e raiz e secadas em estufa com ventilação forçada, a $70^{\circ} \mathrm{C}$, até atingirem peso constante. Em seguida, a massa de matéria seca da parte aérea e da raiz foram separadas e moídas em moinho Willey. Desse material, amostras de $0,1 \mathrm{~g}$ foram retiradas de cada parcela, para a determinação dos teores de $\mathrm{P}\left(\mathrm{mg} \mathrm{g}^{-1}\right)$. Para cada genótipo, foram estimados: massa de matéria seca da planta (MSP); teor de P na planta (PAP), obtido pela média ponderada dos teores na parte aérea e na raiz; a razão entre as matérias secas da parte aérea e raiz (RMS); e o P acumulado (RPA). Além disso, foram estimados os índices de eficiência nutricional de Fageria e de Siddiqi, conforme descritos por Ramos et al. (2009).

A resposta das plantas à disponibilidade do nutriente no solo (RU, $\mathrm{kg} \mathrm{kg}^{-1}$ ) foi determinada pela equação $\mathrm{RU}_{1}=\left(\mathrm{MS}_{\mathrm{se}}-\mathrm{MS}_{\mathrm{ce}}\right) /\left(\mathrm{P}_{\mathrm{se}}-\mathrm{P}_{\mathrm{ce}}\right)$, em que: $\mathrm{MS}_{\mathrm{se}}$ é a massa de matéria seca $(\mathrm{kg})$ produzida na ausência de estresse abiótico (alta disponibilidade de $\mathrm{P}$ ); $\mathrm{MS}_{\mathrm{ce}}$ é a massa de matéria seca produzida sob estresse (baixa disponibilidade de $\mathrm{P}$ ); $\mathrm{P}_{\mathrm{se}}$ é o conteúdo $(\mathrm{kg})$ de $\mathrm{P}$ disponível (Mehlich 1) no solo adubado (sem estresse); e $\mathrm{P}_{\text {ce }}$ é o conteúdo de $\mathrm{P}$ no solo que não recebeu adubação (com estresse).

Com base nos dados do experimento em casa de vegetação, determinaram-se também os índices da eficiência de utilização de P (EUP, $\mathrm{g}^{2} \mathrm{mg}^{-1}$ ) e de translocação do nutriente absorvido para a parte 
aérea (EtP, $\left.\mathrm{kg} \mathrm{kg}^{-1}\right)$, a partir das seguintes fórmulas: EUP $=\left[(\mathrm{MS})^{2}\right] / \mathrm{P}_{\mathrm{ac}}$ e EtP $=\mathrm{P}_{\mathrm{acs}} / \mathrm{P}_{\mathrm{ac}}$, em que MS é a massa de matéria seca, $\mathrm{P}_{\mathrm{ac}}$ é o $\mathrm{P}$ acumulado na planta, e $\mathrm{P}_{\text {acs }}$ é o $\mathrm{P}$ acumulado na parte aérea.

No experimento de campo, foram consideradas duas disponibilidades contrastantes de P: baixa, em que foram aplicados $200 \mathrm{~kg} \mathrm{ha}^{-1}$ da formulação NPK 4-14-8 (28 kg ha-1 de $\mathrm{P}_{2} \mathrm{O}_{5}$ ); e alta, com $800 \mathrm{~kg} \mathrm{ha}^{-1}$ da mesma formulação $\left(112 \mathrm{~kg} \mathrm{ha}^{-1} \mathrm{de} \mathrm{P}_{2} \mathrm{O}_{5}\right.$ ). Para formulação do adubo, foram utilizados como fonte dos nutrientes: ureia, cloreto de potássio e superfosfato simples.

$\mathrm{O}$ delineamento experimental foi o de blocos ao acaso, em esquema fatorial $15 \times 2$, com duas repetições, implantado na Estação Experimental do Aeroporto (204' $\left.38^{\prime \prime} \mathrm{S} ; 42^{\circ} 50^{\prime} 40^{\prime \prime} \mathrm{W}\right)$, da Universidade Federal de Viçosa, no ano agrícola de 2002/2003. A unidade experimental foi constituída por duas linhas de cinco metros, com espaçamento entre plantas de $0,90 \times 0,20 \mathrm{~m}$, que resultou no estande estimado de 55.555 plantas ha-1. Os demais nutrientes, $\mathrm{N}, \mathrm{K}, \mathrm{Ca}, \mathrm{S}, \mathrm{Mg}$ e micronutrientes, foram fornecidos nas condições adequadas para a cultura, de acordo com as recomendações técnicas para a cultura do milho na região. Os caracteres avaliados foram: produtividade de grãos $\left(\mathrm{kg} \mathrm{ha}^{-1}\right.$, corrigido pelo estande final e umidade dos grãos para 13\%) e a resposta das plantas à disponibilidade do nutriente no solo (RU), obtida a partir dos dados de produção de grãos ao invés dos de matéria seca utilizados no experimento anterior.

Os dados foram submetidos à análise de variância e, para os caracteres avaliados em casa de vegetação em que os genótipos apresentaram diferenças significativas a $5 \%$ de probabilidade, foram realizadas análises dialélicas simples e conjunta, para as duas doses de $\mathrm{P}$, utilizando o método 4 proposto por Griffing (1956), tendo-se considerado apenas as gerações $\mathrm{F}_{1}$. $\mathrm{O}$ modelo simples foi representado por:

$$
\mathrm{Y}_{\mathrm{ij}}=\mathrm{m}+\mathrm{g}_{\mathrm{i}}+\mathrm{g}_{\mathrm{j}}+\mathrm{s}_{\mathrm{ij}}+\varepsilon_{\mathrm{ij}}
$$

e o modelo composto por,

$$
\mathrm{Y}_{\mathrm{ij}}=\mathrm{m}+\mathrm{g}_{\mathrm{i}}+\mathrm{g}_{\mathrm{j}}+\mathrm{s}_{\mathrm{jk}}+\mathrm{a}_{\mathrm{k}}+\mathrm{ga}_{\mathrm{ik}}+\mathrm{ga}_{\mathrm{jk}}+\mathrm{as}_{\mathrm{ijk}}+\varepsilon_{\mathrm{ij}}
$$

em que: $\mathrm{Y}_{\mathrm{ij}}$ é o valor médio do híbrido $\mathrm{ij}$; $\mathrm{m}$, a média geral; $\mathrm{g}_{\mathrm{i}}$ e $\mathrm{g}_{\mathrm{j}}$, os efeitos da capacidade geral de combinação dos pais de ordem i e j, respectivamente; $\mathrm{s}_{\mathrm{ij}}$, os efeitos da capacidade específica de combinação para os cruzamentos entre os pais de ordem i e $\mathrm{j} ; \varepsilon_{\mathrm{ij}}$, o erro experimental médio; $\mathrm{a}_{\mathrm{k}}$, o efeito do ambiente $\mathrm{k}$; $\mathrm{ga}_{\mathrm{ik}}$ e $\mathrm{ga}_{\mathrm{jk}}$, os efeitos da interação entre a capacidade geral de combinação (CGC) dos pais de ordem $\mathrm{i}$ e $\mathrm{j}$, respectivamente, com os ambientes $\mathrm{k}$; $\mathrm{as}_{\mathrm{ijk}}$, o efeito da interação entre a capacidade específica de combinação (CEC) entre os pais i e j e o ambiente $\mathrm{k}$.

A fim de identificar características úteis na identificação precoce ou na seleção indireta de genótipos mais eficientes no uso de $\mathrm{P}$, foi realizada a análise de correlação, tendo-se utilizado o coeficiente de Spearman entre os caracteres avaliados em casa de vegetação e em campo. Para a realização de todas as análises, utilizou-se o SAS, versão 9.1 (SAS Institute, 2003).

\section{Resultados e Discussão}

As análises de variância individuais e conjuntas de todos os caracteres avaliados nos experimentos em casa de vegetação e em campo indicaram que as condições de ambiente (A) proporcionadas pelas duas doses de fósforo foram contrastantes. Observaram-se também efeitos significativos das $\mathrm{CH}$ para a maioria dos caracteres avaliados, o que evidencia a existência de variabilidade genética entre os genótipos utilizados. Essa condição possibilitaria ganhos genéticos com a seleção. A significância da interação $\mathrm{CHxA}$, para a maioria dos caracteres, indica que a disponibilidade de $\mathrm{P}$ no solo influenciou de forma diferente $\mathrm{o}$ comportamento das $\mathrm{CH}$.

$\mathrm{Na}$ presença de interação, presume-se que haja possibilidade de realização da seleção em cada condição específica. Os coeficientes de variação das características radiculares apresentaram magnitudes um pouco elevadas em relação a caracteres agronômicos, mas semelhantes aos valores que têm sido relatados em outros trabalhos para caracteres de raiz (Alves et al., 2002; Machado et al., 2004).

Em relação às análises dialélicas, observou-se que, sob alta disponibilidade de P no solo, os efeitos de CEC apresentam maiores magnitudes do que os de CGC, para RMS e RPA (Tabela 1). Sob baixa disponibilidade de $\mathrm{P}$, os efeitos aditivos foram superiores aos não aditivos, para massa de matéria seca da planta e teor de $\mathrm{P}$ na planta. Quanto à RPA, nenhum dos efeitos foi significativo e, para RMS, apenas a CEC foi significativa. Assim, indiferentemente da 
disponibilidade de $\mathrm{P}$ no solo, maiores ganhos deverão ocorrer por meio do uso de estratégias de melhoramento interpopulacionais, de forma a explorar os efeitos não aditivos entre populações e os aditivos dentro de populações (Freitas Júnior et al., 2006). Esses resultados estão de acordo com os obtidos por Chen et al. (2008, 2009), que identificaram QTL's relacionados a EUP, com efeitos, predominantemente, de dominância parcial ou de sobredominância. Coimbra et al. (2008) avaliaram a capacidade de combinação de cultivares de milho para produtividade de grãos sob alta e baixa disponibilidade de fósforo no solo e encontraram efeitos significativos de CGC e CEC, com maior magnitude desse último.

$\mathrm{Na}$ análise dialélica conjunta, a presença da interação significativa CEC x A para MSP e PAP indica que não há consistência entre os efeitos não aditivos em ambas disponibilidades de $\mathrm{P}$, o que indica que a seleção específica nesses ambientes capitalizaria a interação dos efeitos não aditivos x ambientes (Tabela 1). Dessa forma, o controle genético aparentemente é diferenciado nas duas situações. Neste trabalho, os caracteres RMS e RPA não apresentaram significância dessas interações, ou seja, estes caracteres discriminaram os genótipos de forma semelhante nas diferentes disponibilidades de P. Para os caracteres MSP e PAP, no entanto, a interação foi significativa. Observou-se que, para MSP sob baixa disponibilidade de $\mathrm{P}$, as $\mathrm{CH}$ que apresentaram os maiores valores de CEC foram 1x6, 3x5, 4x6 e 4x5
(Tabela 2). Entretanto, sob alta disponibilidade de P, as melhores $\mathrm{CH}$ foram $1 \times 2,3 \times 6$ e $2 \times 6$. Para PAP, os maiores valores de $\mathrm{CEC}$ foram observados nas $\mathrm{CH} 3 \mathrm{x} 6$ e $1 \times 5$ em baixa disponibilidade de $\mathrm{P}$ no solo e as $\mathrm{CH}$ $1 \times 6,3 \times 5$ e $4 \times 6$ em alta disponibilidade de $P$.

Neste trabalho, as $\mathrm{CH} 2 \times 5,3 \times 5$ e 4x6 apresentaram valores positivos de CEC para MSP e PAP, em baixa e alta disponibilidade de $\mathrm{P}$. O genótipo DKB 747 (5) está presente na composição de duas dessas combinações, o que é um indicativo de que esse genótipo, quando usado como genitor, tende a produzir híbridos com maior heterose para estes dois caracteres, independentemente da disponibilidade de P no solo. Isto se deve, possivelmente, ao fato de o genótipo DKB 747 apresentar características radiculares que permitem maior adaptação a solos de cerrado.

Para o desenvolvimento de híbridos, além de altas CEC nas combinações híbridas, recomenda-se que haja participação no cruzamento de, no mínimo, um genitor com elevada CGC (Betrán et al., 2003). Entre os genitores com alta CGC, tanto para MSP como para PAP, destacou-se o AG 9010 (3), que participou da formação das $\mathrm{CH} 3 \times 6$ e $3 \times 5$, as quais figuraram entre as superiores nas duas disponibilidade de $\mathrm{P}$. Com isso, ao se considerar os valores de CEC e CGC, constata-se que a combinação entre linhagens derivadas do AG 9010 (3) com as do DKB 747 (5) possivelmente produzem $\mathrm{CH}$ com maiores capacidades produtivas e tolerâncias ao estresse por deficiência de $\mathrm{P}$ no solo.

Tabela 1. Quadrados médios das análises dialélicas individual e conjunta para massa de matéria seca da planta (MSP), teor de P na planta (PAP), razão entre a matéria seca da parte aérea e da raiz (RMS) e P acumulado (RPA) em genótipos de milho cultivados no experimento em casa de vegetação.

\begin{tabular}{|c|c|c|c|c|c|c|c|c|c|}
\hline \multirow[t]{2}{*}{ Fonte de variação ${ }^{(1)}$} & \multirow[t]{2}{*}{ GL } & \multicolumn{2}{|c|}{ MSP } & \multicolumn{2}{|c|}{ PAP } & \multicolumn{2}{|c|}{ RMS } & \multicolumn{2}{|c|}{ RPA } \\
\hline & & Alto P & Baixo P & Alto P & Baixo P & Alto P & Baixo P & Alto P & Baixo P \\
\hline & \multicolumn{9}{|c|}{ Análise dialélica individual } \\
\hline $\mathrm{CH}$ & 14 & $024,87 * *$ & $055,36 * *$ & $32,16 * *$ & $51,01 * *$ & $0,034^{*}$ & $0,034 * *$ & $0,128 * *$ & $0,137^{\text {ns }}$ \\
\hline $\mathrm{CGC}$ & 05 & $040,20 * *$ & $102,87 * *$ & $46,33 * *$ & $93,60 * *$ & $0,026^{\mathrm{ns}}$ & $0,057 * *$ & $0,046^{\mathrm{ns}}$ & $0,123^{\text {ns }}$ \\
\hline CEC & 09 & $016,35 * *$ & $028,96 * *$ & $24,30 * *$ & $27,34 * *$ & $0,038 *$ & $0,021^{\mathrm{ns}}$ & $0,173 * *$ & $0,144^{\mathrm{ns}}$ \\
\hline Resíduo & 22 & 003,81 & 007,04 & 03,31 & 08,23 & 0,014 & 0,013 & 0,037 & 0,094 \\
\hline \multirow[t]{2}{*}{$\mathrm{CV}(\%)$} & & 6,54 & 12,18 & 7,00 & 15,20 & 13,00 & 13,26 & 14,91 & 28,39 \\
\hline & \multicolumn{9}{|c|}{ Análise dialélica conjunta } \\
\hline $\mathrm{CH}$ & 14 & \multicolumn{2}{|c|}{$66,46 * *$} & \multicolumn{2}{|c|}{$65,73 * *$} & \multicolumn{2}{|c|}{$0,036^{\mathrm{ns}}$} & \multicolumn{2}{|c|}{$0,195 *$} \\
\hline $\mathrm{CGC}$ & 5 & \multicolumn{2}{|c|}{$134,03 * *$} & \multicolumn{2}{|c|}{$123,82 * *$} & \multicolumn{2}{|c|}{$0,031^{\mathrm{ns}}$} & \multicolumn{2}{|c|}{$0,121^{*}$} \\
\hline $\mathrm{CEC}$ & 9 & \multicolumn{2}{|c|}{$28,92^{\mathrm{ns}}$} & \multicolumn{2}{|c|}{$33,46^{\mathrm{ns}}$} & \multicolumn{2}{|c|}{$0,038^{\text {ns }}$} & \multicolumn{2}{|c|}{$0,235^{*}$} \\
\hline Ambiente (A) & 1 & \multicolumn{2}{|c|}{$1.000,10 * *$} & \multicolumn{2}{|c|}{$889,90 * *$} & \multicolumn{2}{|c|}{$0,030 * *$} & \multicolumn{2}{|c|}{$0,471 * *$} \\
\hline $\mathrm{CHxA}$ & 14 & \multicolumn{2}{|c|}{$13,76 * *$} & \multicolumn{2}{|c|}{$17,44 * *$} & \multicolumn{2}{|c|}{$0,032 *$} & \multicolumn{2}{|c|}{$0,070^{\mathrm{ns}}$} \\
\hline CGCxA & 5 & \multicolumn{2}{|c|}{$9,03^{\mathrm{ns}}$} & \multicolumn{2}{|c|}{$16,11 *$} & \multicolumn{2}{|c|}{$0,051 * *$} & \multicolumn{2}{|c|}{$0,048^{\mathrm{ns}}$} \\
\hline CECXA & 9 & \multicolumn{2}{|c|}{$16,39 * *$} & \multicolumn{2}{|c|}{$18,18 * *$} & & $21^{\mathrm{ns}}$ & & \\
\hline Resíduo & 46 & 5, & & & & & & & \\
\hline CV $(\%)$ & & 9, & & 10 , & & & & & \\
\hline
\end{tabular}

${ }^{(1)} \mathrm{CH}$, combinações híbridas; CGC, capacidade geral de combinação e CEC, capacidade especifica de combinação. ${ }^{\text {ns}}$ Não significativo. * e **Significativo a 5 e $1 \%$ de probabilidade, respectivamente, pelo teste $\mathrm{F}$. 
Foi observada correlação significativa e positiva entre as EUP em baixa e alta disponibilidade de $\mathrm{P}$ (Tabela 3), o que indica que as plantas que são eficientes na translocação do $\mathrm{P}$ em baixas disponibilidades também o são em altas disponibilidades. Estes dois caracteres também apresentaram associações altamente significativas com PAP, sob alta disponibilidade de $\mathrm{P}$, e com MSP, nos dois níveis estudados. Esses resultados estão de acordo com Machado et al. (2001) que, ao avaliar variedades de milho em três disponibilidades de $\mathrm{P}$ em casa de vegetação e campo, observaram que, em casa de vegetação, as correlações entre produtividade de grãos e eficiência de absorção de fósforo foram positivas e significativas e que as variedades mais produtivas foram as que apresentaram maior massa de matéria seca.

É provável que genes diferentes estejam envolvidos no controle da EUP, nas diferentes disponibilidades de P. Chen et al. (2009) não identificaram QTL's

Tabela 2. Capacidades geral e específica de combinação para massa seca da planta (MSP), teor de P na planta (PAP), razão entre a matéria seca da parte aérea e da raiz (RMS) e P acumulado (RPA), em genótipos de milho e suas combinações híbridas $(\mathrm{CH})$ cultivados em experimento de casa de vegetação.

\begin{tabular}{|c|c|c|c|c|c|c|c|c|}
\hline \multirow[t]{2}{*}{ Genótipo } & \multicolumn{2}{|c|}{ MSP } & \multicolumn{2}{|c|}{ PAP } & \multicolumn{2}{|c|}{ RMS } & \multicolumn{2}{|c|}{ RPA } \\
\hline & Alto P & Baixo P & Alto P & Baixo P & Alto P & Baixo P & Alto P & Baixo P \\
\hline & \multicolumn{8}{|c|}{ Capacidade específica de combinação } \\
\hline $\mathrm{CH} 1 \times 2$ & 0,129 & 2,761 & 0,912 & $-0,621$ & $-0,020$ & 0,047 & 0,321 & 0,309 \\
\hline $\mathrm{CH} 1 \times 3$ & $-0,076$ & 1,577 & $-1,942$ & $-2,111$ & $-0,089$ & $-0,070$ & $-0,170$ & $-0,040$ \\
\hline $\mathrm{CH} 1 \times 4$ & $-1,546$ & $-0,548$ & 1,680 & $-2,631$ & $-0,005$ & 0,007 & $-0,089$ & $-0,249$ \\
\hline $\mathrm{CH} 1 \times 5$ & $-1,355$ & 0,272 & 2,284 & 0,824 & 0,111 & 0,216 & 0,186 & 0,277 \\
\hline $\mathrm{CH} 1 \times 6$ & 2,848 & $-4,063$ & $-2,933$ & 4,539 & 0,003 & $-0,201$ & $-0,249$ & $-0,298$ \\
\hline $\mathrm{CH} 2 \times 3$ & $-0,218$ & $-6,266$ & $-1,869$ & $-0,537$ & 0,178 & $-0,068$ & 0,222 & $-0,094$ \\
\hline $\mathrm{CH} 2 \times 4$ & 0,625 & $-1,185$ & $-0,532$ & 1,622 & 0,043 & 0,082 & $-0,009$ & 0,082 \\
\hline $\mathrm{CH} 2 \times 5$ & 0,615 & 2,315 & 1,267 & 1,590 & $-0,168$ & $-0,104$ & $-0,164$ & $-0,316$ \\
\hline $\mathrm{CH} 2 \times 6$ & $-1,151$ & 2,375 & 0,223 & $-2,054$ & $-0,033$ & 0,042 & $-0,370$ & 0,019 \\
\hline $\mathrm{CH} 3 \times 4$ & $-2,002$ & $-0,822$ & $-3,507$ & $-1,870$ & $-0,019$ & 0,089 & $-0,044$ & 0,356 \\
\hline $\mathrm{CH} 3 \times 5$ & 2,387 & 0,283 & 0,029 & 3,934 & $-0,027$ & $-0,065$ & $-0,073$ & $-0,242$ \\
\hline $\mathrm{CH} 3 \times 6$ & $-0,091$ & 5,228 & 7,289 & 0,584 & $-0,043$ & 0,113 & 0,065 & 0,019 \\
\hline $\mathrm{CH} 4 \times 5$ & 1,441 & 1,612 & 1,679 & $-0,200$ & $-0,003$ & $-0,137$ & $-0,179$ & $-0,083$ \\
\hline $\mathrm{CH} 4 \times 6$ & 1,485 & 0,942 & 0,680 & 3,079 & $-0,015$ & $-0,042$ & 0,321 & $-0,106$ \\
\hline \multirow[t]{2}{*}{ CH 5x6 } & $-3,088$ & $-4,482$ & 5,258 & 6,148 & 0,088 & 0,088 & 0,231 & 0,365 \\
\hline & \multicolumn{8}{|c|}{ Capacidade geral de combinação } \\
\hline Sol da manhã (1) & $-0,749$ & $-1,786$ & $-1,879$ & 0,900 & $-0,038$ & 0,066 & $-0,061$ & 0,050 \\
\hline BR $106(2)$ & $-1,563$ & $-4,846$ & $-4,770$ & $-3,781$ & 0,099 & $-0,004$ & 0,229 & 0,075 \\
\hline AG $9010(3)$ & 1,888 & 6,002 & 5,414 & 2,332 & 0,033 & 0,009 & $-0,013$ & 0,004 \\
\hline BR 201 (4) & 0,961 & 0,196 & 0,160 & $-0,228$ & 0,043 & $-0,102$ & $-0,009$ & $-0,123$ \\
\hline DKB 747 (5) & $-0,952$ & $-0,637$ & $-0,536$ & $-1,619$ & 0,008 & 0,034 & $-0,003$ & 0,049 \\
\hline Caiano (6) & 0,415 & 1,071 & 1,610 & 2,395 & $-0,146$ & $-0,003$ & $-0,042$ & $-0,055$ \\
\hline
\end{tabular}

Tabela 3. Correlações entre os caracteres avaliados em casa de vegetação - resposta à disponibilidade de $\mathrm{P}\left(\mathrm{RU}_{1}\right)$, eficiência de translocação de P em alta (EtAP) e baixa disponibilidade de P (EtBP), eficiência de utilização de P em alta (EuAP) e baixa disponibilidade de $\mathrm{P}$ (EuBP), teor de $\mathrm{P}$ na planta em alta P (PAPAP) e baixa disponibilidade de P (PAPBP), massa seca da planta em alta (MSPAP) e baixa disponibilidade de $\mathrm{P}$ (MSPBP) -, e em campo - resposta à disponibilidade de $\mathrm{P}\left(\mathrm{RU}_{2}\right)$ e produtividade de grãos sob alta (PGAP) e baixa disponibilidade de P (PGBP) -, para os 21 genótipos de milho avaliados (6 parentais e 15 combinações híbridas).

\begin{tabular}{|c|c|c|c|c|c|c|c|c|c|c|c|}
\hline Caractere & $\mathrm{RU}_{1}$ & $\mathrm{RU}_{2}$ & EtAP & EtBP & EuAP & EuBP & PAPAP & PAPBP & MSPAP & MSPBP & PGAP \\
\hline $\mathrm{RU}_{2}$ & $-0,08^{\mathrm{ns}}$ & & & & & & & & & & \\
\hline EtAP & $-0,45^{*}$ & $-0,22^{\mathrm{ns}}$ & & & & & & & & & \\
\hline EtBP & $0,38^{\mathrm{ns}}$ & $-0,29^{\mathrm{ns}}$ & $0,09^{\text {ns }}$ & & & & & & & & \\
\hline EuAP & $-0,07^{\mathrm{ns}}$ & $0,45^{*}$ & $-0,18^{\mathrm{ns}}$ & $-0,17^{\mathrm{ns}}$ & & & & & & & \\
\hline EuBP & $0,66^{* *}$ & $0,16^{\mathrm{ns}}$ & $-0,29^{\mathrm{ns}}$ & $0,14^{\mathrm{ns}}$ & $0,54 * *$ & & & & & & \\
\hline PAPAP & $0,39^{\mathrm{ns}}$ & $0,34^{\mathrm{ns}}$ & $-0,33^{\mathrm{ns}}$ & $-0,09^{\mathrm{ns}}$ & $0,81 * *$ & $0,68 * *$ & & & & & \\
\hline PAPBP & $-0,11^{\mathrm{ns}}$ & $0,40^{\mathrm{ns}}$ & $-0,24^{\mathrm{ns}}$ & $-0,24^{\mathrm{ns}}$ & $0,94 * *$ & $0,41^{\mathrm{ns}}$ & $0,79 * *$ & & & & \\
\hline MSPAP & $0,66^{* *}$ & $0,16^{\mathrm{ns}}$ & $-0,41^{\mathrm{ns}}$ & $0,05^{\mathrm{ns}}$ & $0,63 * *$ & $0,90 * *$ & $0,88 * *$ & $0,58 * *$ & & & \\
\hline MSPBP & $-0,07^{\mathrm{ns}}$ & $0,45^{*}$ & $-0,18^{\mathrm{ns}}$ & $-0,17^{\mathrm{ns}}$ & $0,99 * *$ & $0,54 * *$ & $0,81 * *$ & $0,94 * *$ & $0,64 * *$ & & \\
\hline PGAP & $-0,16^{\mathrm{ns}}$ & $0,79 * *$ & $-0,01^{\mathrm{ns}}$ & $-0,25^{\mathrm{ns}}$ & $0,53 * *$ & $0,29^{\mathrm{ns}}$ & $0,37^{\mathrm{ns}}$ & $0,42^{\mathrm{ns}}$ & $0,20^{\mathrm{ns}}$ & $0,53 * *$ & \\
\hline PGBP & $0,15^{\mathrm{ns}}$ & $-0,35^{\mathrm{ns}}$ & $0,36^{\mathrm{ns}}$ & $0,12^{\mathrm{ns}}$ & $0,16^{\mathrm{ns}}$ & $0,18^{\text {ns }}$ & $0,26^{\text {ns }}$ & $0,19^{\text {ns }}$ & $0,26^{\mathrm{ns}}$ & $0,16^{\mathrm{ns}}$ & $-0,13^{\mathrm{ns}}$ \\
\hline
\end{tabular}

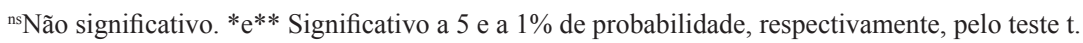


coincidentes relacionados à EUP sob diferentes níveis de disponibilidade do nutriente no solo. Este fato reitera a possibilidade de se realizar seleção específica nas diferentes disponibilidades de fósforo. Para realização da seleção específica, tanto o acúmulo de $\mathrm{P}$ como o de matéria seca são caracteres de grande utilidade para a seleção precoce de genótipos mais eficientes na utilização do P.

As correlações positivas observadas entre resultados obtidos em casa de vegetação e os de campo permitem ao pesquisador utilizar o cultivo em ambientes protegidos para avaliação da EUP, o que reduziria o tempo e o trabalho na seleção inicial dos genótipos (Machado et al., 2004). As eficiências de translocação, RU em casa de vegetação e a PG a campo em baixa disponibilidade de $\mathrm{P}$ não apresentaram associações significativas com nenhum outro caráter (Tabela 3), o que indica que não devem ser recomendados para seleção indireta.

A EUP em baixa disponibilidade de P apresentou correlação altamente significativa com RU em campo, o que indica que plantas que são mais eficientes na utilização do $\mathrm{P}$, em baixas disponibilidades do nutriente no solo, são mais responsivas a adubações fosfatadas a campo (Tabela 3). Por sua vez, a RU a campo, assim como a EUP em baixa disponibilidade de P, em casa de vegetação, está altamente correlacionada com a PG em altas disponibilidades de P no solo. Desse modo, ao se realizar a seleção de genótipos quanto à EUP em casa de vegetação, sob baixa disponibilidade de $\mathrm{P}$, seriam selecionados genótipos mais produtivos a campo em situações normais de cultivo. Entretanto, ressalta-se que os genótipos com elevada EUP podem ser ineficientes no aproveitamento interno do $\mathrm{P}$, em razão de uma possível baixa capacidade de translocação de fotoassimilados na planta, que resultaria em grande quantidade de massa de matéria seca, mas não em elevada produção de grãos.

\section{Conclusões}

1. Os efeitos não aditivos têm maior importância para caracteres relacionados à eficiência da utilização do fósforo, de modo que a seleção deve ser realizada nas combinações híbridas.

2. O acúmulo de fósforo e de matéria seca são caracteres que podem ser utilizados na seleção indireta de plantas mais eficientes na utilização do $\mathrm{P}$, sob alta e baixa disponibilidade de P no solo.

\section{Referências}

ALVES, V.M.C.; PARENTONI, S.N.; VASCONCELLOS, C.A.; PITTA, G.V.E.; FRANÇA, C. de M. Cinética de absorção de fósforo e crescimento do sistema radicular de genótipos de milho contrastantes para eficiência a fósforo. Revista Brasileira de Milho e Sorgo, v.1, p.85-92, 2002.

BALIGAR, V.C.; FAGERIA, N.K. Plant nutrient efficiency: towards the second paradigm. In.: SIQUEIRA, J.O.; MOREIRA, F.M.S.; LOPES, A.S.; GUILLHERME, L.R.G.; FAQUIM, V.; FURTINI NETO, A.E.; CARVALHO, J.G. (Ed.). Inter-relação fertilidade, biologia do solo e nutrição de plantas. Lavras: SBSC: UFLA, 1999. p.183-204.

BETRÁN, F.J.; BECK, D.; BÄNZIGER, M.; EDMEADES, G.O. Genetic analysis of inbred and hybrid grain yield under stress and nonstress environments in tropical maize. Crop Science, v.43, p.807-817, 2003.

CHEN, J.; XU, L.; CAI, Y.; XU, J. Identification of QTLs for phosphorus utilization efficiency in maize (Zea mays L.) across $\mathrm{P}$ levels. Euphytica, v.167, p.245-252, 2009.

CHEN, J.; XU, L.; CAI, Y.; XU, J. QTL mapping of phosphorus efficiency and relative biologic characteristics in maize (Zea mays L.) at two sites. Plant and Soil, v.313, p.251-266, 2008.

COIMBRA, R.R.; MARTINS, E.C.A.; MIRANDA, G.V.; NAOE, L.K.; CARDOSO, E.A.; ARCHANGELO, E.R. Capacidade de combinação de genótipos de milho para solos com baixos níveis de fertilidade. Revista de Ciências Agrárias, n.50, p.23-33, 2008.

FREITAS JÚNIOR, S. de P.; AMARAL JÚNIOR, A.T. do; PEREIRA, M.G.; CRUZ, C.D.; SCAPIM, C.A. Capacidade combinatória em milho-pipoca por meio de dialelo circulante. Pesquisa Agropecuária Brasileira, v.41, p.1599-1607, 2006.

GAUME, A.; MÃCHLER, F.; DE LEÓN, C.; NARRO, L.; FROSSARD, E. Low-P tolerance by maize (Zea mays L.) genotypes: significance of root growth, and organic acids and acid phosphatase root exudation. Plant and Soil, v.228, p.253-264, 2001.

GRIFFING, B. Concept of general and specific and specific combining ability in relation to diallel crossing systems. Australian Journal of Biological Sciences, v.9, p.463-493, 1956.

HAMMOND, J.P.; BROADLEY, M.R.; WHITE, P.J. Genetic responses to phosphorus deficiency. Annals of Botany, v.94, p.323-332, 2004.

HIREL, B.; LE GOUIS, J.; NEY, B.; GALLAIS, A. The challenge of improving nitrogen use efficiency in crop plants: towards a more central role for genetic variability and quantitative genetics within integrated approaches. Journal of Experimental Botany, v.587, p.2369-2387, 2007.

MACHADO, C.T. de T.; FURLANI, A.M.C.; MACHADO, A.T. Índices de eficiência de variedades locais e melhoradas de milho ao fósforo. Bragantia, v.60, p.225-238, 2001.

MACHADO, C.T. de T.; MACHADO, A.T.; FURLANI, A.M.C. Variação intrapopulacional em milho para características relacionadas com a eficiência de absorção e utilização de fósforo. Revista Brasileira de Milho e Sorgo, v.3, p.77-91, 2004. 
MIRANDA, G.V.; GODOY, C.L.; GALVÃO, J.C.C.; SANTOS, I.C. dos; ECKERT, F.R.; SOUZA, L.V. de. Selection of discrepant maize genotypes for nitrogen use efficiency by a chlorophyll meter. Crop Breeding and Applied Biotechnology, v.5, p.451-459, 2005.

MIRANDA, G.V.; SOUZA, L.V.; GALVÃO, J.C.C.; GUIMARÃES, L.J.M.; VAZ DE MELO, A.; SANTOS, I.C. Genetic variability and heterotic groups of Brazilian popcorn populations. Euphytica, v.162, p.431-440, 2008.

OLIVEIRAJUNIOR,A.; MIRANDA, G.V.; CRUZ, C.D. Prediction of F3 populations based on unbalanced diallel crossing systems. Pesquisa Agropecuária Brasileira, v.34, p.781-787, 1999.

PARENTONI, S.N.; SOUZA JUNIOR, C.L. de. Phosphorus acquisition and internal utilization efficiency in tropical maize genotypes. Pesquisa Agropecuária Brasileira, v.43, p.893-901, 2008.
RAMOS, S.J.; FAQUIN, V.; RODRIGUES C.R.; SILVA, C.A.; BOLDRIN, P.F. Biomass production and phosphorus use of forage grasses fertilized with two phosphorus sources. Revista Brasileira de Ciência do Solo, v.33, p.335-343, 2009.

SAS INSTITUTE. SAS/STAT: user's guide. Version 9.1. Cary: SAS Institute, 2003.

SOUZA, L.V. de; MIRANDA, G.V.; GALVÃO, J.C.C.; ECKERT, F.R.; MANTOVANI, E.E.; LIMA, R.O.; GUIMARÃES, L.J.M. Genetic control of grain yield and nitrogen use efficiency in tropical maize. Pesquisa Agropecuária Brasileira, v.43, p.1517-1523, 2008.

YUN, S.J.; KAEPPLER, S.M. Induction of maize acid phosphatase activities under phosphorus starvation. Plant and Soil, v.237, p.109-115, 2001.

Recebido em 20 de agosto de 2009 e aprovado em 5 de abril de 2010 